Research Article

\title{
Accuracy of ultrasound guided versus blind knee intra-articular injection for knee osteoarthritis prolotherapy
}

\begin{abstract}
Introduction: Knee osteoarthritis (OA) is one of the most common joint disabling diseases among adult patients. Ultrasound has been recommended for intra-articular injection, however its accuracy only been compared to blind approach in expert and inexpert clinicians.

Objective: To compare the accuracy of intra-articular injection under ultrasound guide versus blind injection by expert and inexpert clinician.

Setting: Outpatient; adults with mild-to-severe knee OA.

Participants: Adults with at least 3 months of symptomatic KOA, recruited from clinical and community settings, participated in the study.

Methods: Total of 220 patients with knee osteoarthritis were enrolled from which 100 patients were in injected under ultrasound guided and 123 patients injected blindly by supralateral approach. Then patients were divided into two groups, group A was injected by an expert pain physician and group B with an inexpert physician. The accuracy was determined using fluoroscopy at the same time of injection.

Results: In group A (expert), failed intra-articular injection was confirmed by fluoroscopy in $2 \%$ from 50 patient in ultrasound guided injection and $4.26 \%$ from 47 patients in blind injection but no significant difference between blind and ultrasound injection when it was performed by highly expert clinician $(\mathrm{p}=0.61)$. In group B (inexpert), failure in intraarticular injection was in confirmed by fluoroscopy in $6 \%$ from 50 patient in ultrasound guided injections and $16(21 \%)$ from 76 patients in blind injections; and there was significant difference between blind and ultrasound guided injection when it was performed by inexpert clinician $(\mathrm{p}=0.02)$.
\end{abstract}

Conclusion: ultrasound improves accuracy of knee intra-articular injection independent of clinician level of expertise. Blind approaches compose a high failure rate particularly when performed by inexpert clinicians.

Keywords: ultrasound, osteoarthritis, knee, intra-articular
Volume 5 Issue 2 - 2016

\author{
Seyed Masoud Hashemi, Behnam Hosseini, \\ Mehdi Zhand, Reza Pourrosta \\ Research Center of Shohada Tajrish Hospital, Shahid Beheshti \\ Medical University, Iran
}

Correspondence: Behnam Hosseini, Research Center of Shohada Tajrish Hospital, Shahid Beheshti Medical University, Iran,Email dr_b_hosseini@yahoo.com

Received: June 03, 2016 | Published: July 13, 2016

\section{Introduction}

Knee osteoarthritis (OA) is one of the most common joint disabling diseases among adult patients. Symptomatic knee OA occurs in $10 \%$ men and $13 \%$ in women aged 60 years or older but is likely to increase due to the aging of the population and the obesity epidemic. ${ }^{1}$ Osteoarthritis (OA) is characterized by progressive loss of articular cartilage, subchondral bone sclerosis, osteophyte formation, changes in the synovial membrane, and an increased volume of synovial fluid with reduced viscosity. ${ }^{2}$ It is a complex disease with unknown etiology but evidence is growing for the role of systemic factors (such as genetics, dietary intake, estrogen use, and bone density) and of local biomechanical factors (such as muscle weakness, obesity, and joint laxity). ${ }^{3}$ Current mainstay of microinvasive therapy is prolotherapy by intra-articular injections. Prolotherapy with dextrose and with prolozone resulted in the same pain relief or functional improvement in patients with mild to moderate knee OA. ${ }^{4} \mathrm{~A}$ randomized controlled trial (RCT) reported significant improvement in knee OA pain scores when treated with prolotherapy. ${ }^{5}$ Prolotherapy results in progressive improvement of knee pain, function and stiffness in long-term followups. ${ }^{6}$ Blind intra-articular injection is still the choice of injections for many clinicians with acceptable success rate. ${ }^{7}$ However, it could result in extra-articular approach in some patients. Besides, accidental collision of the needle tip with the superolateral pole of the patella during attempted aspiration or injection of the knee joint could led to pain, as well as damage to the chondral cartilage of the patella. ${ }^{8}$ Although, ultrasound has been recommended for intra-articular injection, however its accuracy only been tested on cadaver.

\section{Objective}

To compare the accuracy of intra-articular injection under ultrasound guide versus blind injection by expert and inexpert clinician.

\section{Methods}

\section{Ethics declaration}

The study was reviewed and approved by the university Ethics Committee and been performed in accordance with the ethical standards. Information about the study was given comprehensively both orally and in written form to all patients or their accompanying 
adult. They gave their informed written consents prior to their inclusion in the study.

\section{Patient selection}

Total of 220 patients with knee osteoarthritis were enrolled from which 100 patients were in injected under ultrasound guided and 123 patients injected blindly by supralateral approach. Then patients were divided into two groups, group A was injected by an expert pain physician and group B with an inexpert physician. Patients were recruited from pain clinic of our hospital. They were randomly assigned to be injected under ultrasound guided or not (blind injection). Physical examination was conducted by the lead physician (DR) and radiology was performed by the same radiologist. Inclusion criteria were Patient with osteoarthritis diagnosis based on ACR definition (age $>50$ years, crepitating or morning stiffness less than 35 minutes and radiologic findings), and having symptoms more than 3 month. Exclusion criteria were diabetes, other causes of arthritis such as gout or rheumatoid arthritis, history of surgery or fracture in the knee, history of prolotherapy in past year, or injection of corticosteroid or hyaloronic acid, contraindication to injection including thrombocytopenia, or bleeding diasthasis, severe effusion of knee, and infection of local skin.

\section{Hyaloronic acid}

Hyaloronic acid was injected into the knee intra-articular joint. It is a mixture Glucoronic acid and $\mathrm{N}$-acetyl-glucosamin with a molecular weight of 500-750 KD. It provides visco elasticity of articular fluid and also triggers endogenous hyaloronic production.

\section{Supralateral blind approach}

This technique was performed with the patient supine and the knee extended. This technique aims for the suprapatellar pouch, and allows the needle to pass underneath the articular surface of the patella.

\section{Ultrasound guided injection}

In ultrasound group, all intra-articular injections were performed under ultrasound guide. Ultrasound approach was performed via transverse image and out-of-plain to the knee.

\section{Fluroroscopy}

The accuracy of success or failed intra-articular injection was confirmed by fluoroscopy. After needle insertion, $1 \mathrm{ml}$ of contrast media was injected and then after fluoroscopy and if place of needle was confirmed then hyaloronic acid $(4 \mathrm{ml})$ was also used to inject. Patients underwent fluoroscopy at the same time of injection.

\section{Results}

Total of 220 patients with knee osteoarthritis were enrolled from which 100 patients were in injected under ultrasound guided and 123 patients injected blindly by supralateral approach. Age, sex and BMI of patients were not significantly different between two groups of study (Table 1). Grade of osteoarthritis was not also significantly different between two groups of study.

\section{Expert vs. inexpert physician}

Patients were divided into two groups, group A, who were injected by an expert pain physician and group B injected by an inexpert physician. In group A, from 97 patients, 50 patients $(51.5 \%)$ were injected under ultrasound guide and $47(48.5 \%)$ had blind injection. 29 patients were male, and $68(70 \%)$ were female. Sixty one patients had OA grade 2 and 36 (37\%) had grade 3 OA. Mean age was 63.2 \pm 7.48 years old.

Table I Demographic variables of patients in ultrasound and blind group

\begin{tabular}{llll}
\hline & Ultrasound (1 00) & Blind ( I 23) & p-Value \\
\hline Age & $64.46 \pm 7.16$ & $63.68 \pm 7.54$ & 0.13 \\
Sex (male/female) & $27 / 73$ & $33 / 90$ & 0.55 \\
BMI & $26.67 \pm 3.06$ & $26.88 \pm 2.64$ & 0.58 \\
Grade of Osteoarthritis & & & \\
Grade 2 & 46 & 58 & 0.48 \\
Grade 3 & 54 & 65 & \\
Symptom duration & $6.03 \pm 2.44$ & $6.51 \pm 3.19$ & 0.2 \\
\hline
\end{tabular}

\section{Group A (highly expert)}

In group A, 50 patients had ultrasound guided injections, 14 male and 36 female, 28 had grade 2 and 22 had grade 3 OA. Duration of symptoms was $6.4 \pm 2.23$ and BMI was $27.3 \pm 3.38$. In group A, 47 patient had blind injection by an expert, 15 were male (31.9\%) and 32 were female $(68.1 \%), 33$ patients $(70.3 \%)$ had grade 2 and 14 patients $(29.7 \%)$ had grade 3 OA. There were no significant differences in age, symptom duration, and BMI of patients who were injected by highly expert clinician (group A) using ultrasound or blind approach (Table 2). There were no significant differences in sex and grade of OA of patients who were injected by highly expert clinician (group A) using ultrasound or blind approach (Table 2). In group A, failed intraarticular injection was confirmed by fluoroscopy in $1(2 \%)$ from 50 patient in ultrasound guided injection and $2(4.26 \%)$ from 47 patients in blind injection; and there was no significant difference between blind and ultrasound injection when it was performed by highly expert clinician $(\mathrm{p}=0.61)$. All three failed cases were female and grade 2 which were not significantly different between two groups.

Table 2 Age, symptom duration, BMI, and grade of OA of patients who were injected by highly expert clinicians (group A) using ultrasound or blind approach

\begin{tabular}{llll}
\hline Variables & Ultrasound (50) & Blind (47) & p-Value \\
\hline Age & $65.5 \pm 7.25$ & $63.6 \pm 7.6$ & 0.2 \\
Symptom duration & $6.04 \pm 2.33$ & $6.85 \pm 3.92$ & 0.22 \\
BMI & $27.48 \pm 3.38$ & $27.31 \pm 2.55$ & 0.78 \\
Grade 2 (male/female) & I8-Oct & $21-\mathrm{Dec}$ & 0.2 \\
Grade 3 (male/female) & I8-Apr & II-Mar & 0.12 \\
\hline
\end{tabular}

Table 3 Age, symptom duration, BMI, and grade of OA of patients who were injected by inexpert clinicians (group B) using ultrasound or blind approach

\begin{tabular}{llll}
\hline Variables & Ultrasound (50) & Blind (76) & p-Value \\
\hline Age & $63.36 \pm 6.94$ & $63.72 \pm 7.52$ & 0.78 \\
Symptom Duration & $6.02 \pm 2.58$ & $6.30 \pm 2.65$ & 0.56 \\
BMI & $25.86 \pm 2.50$ & $26.62 \pm 2.65$ & 0.11 \\
Grade 2 (Male/Female) & I2-Jun & I8-Jul & 0.18 \\
Grade 3 (Male/Female) & 25-Jul & Nov-40 & 0.15 \\
\hline
\end{tabular}

\section{Group B (inexpert)}

In group $B$ who were injected by inexpert clinician, 126 patients were enrolled with mean age of $63.58 \pm 7.27$ years old, BMI of $26.32 \pm 2.62$, mean symptom duration of $6.19 \pm 2.62$. From 126 patients, $31(24.6 \%)$ were male and $95(75.4 \%)$ were female, and $43(34.1 \%)$ were grade 2 and $83(65.9 \%)$ were grade $3 \mathrm{OA}$. In this group 50 patients were injected under ultrasound guide and 76 were injected blindly. There were no significant differences in age, symptom duration, and BMI, and grade of OA of patients who were injected by inexpert clinician (group B) using ultrasound or blind approach (Table 3). In group B (inexpert), failure in intra-articular injection was in confirmed by 
fluoroscopy in $3(6 \%)$ from 50 patient in ultrasound guided injections and $16(21 \%)$ from 76 patients in blind injections; and there was significant difference between blind and ultrasound guided injection when it was performed by inexpert clinician $(p=0.02)$. In group $B$, from 19 failed cases, 15 cases were female and 4 were male; 4 cases were grade 2 and 15 cases were grade 3 which were not significantly different between two groups.

In this study we demonstrated the role of ultrasound in improving success rate of knee intra-articular injection even in hand of inexpert clinician. Several studies have used various materials and compared their effect in healing signs and symptoms of knee OA but it seems that technique of intra-articular injection is also of importance. Various blind techniques have been attempted in recent years with different success rates. Hermans et al. ${ }^{9}$ determined that the superolateral approach results in the highest pooled accuracy rate of $91 \%$ (95\% CI 84\%-99\%), although this approach still results in a substantial amount of extra-articular needle placements. ${ }^{9}$ Recently, researches have focused on various equipments and approaches for improvement of knee intra-articular injection. Ultrasound has been applied in recent years to determine the anatomy of articular spaces and increasing the success rate of injections. Other applied equipments such as fluoroscopy or MRI have limitations which makes ultrasound a better choice for knee intra-articular injections. Ultrasound is a safe equipment but its ability to improve success rate has not been studied, and besides many clinician may find themselves of no need to ultrasound assistant. Here we demonstrated how ultrasound improved intra-articular technique success rate even in the hands of inexpert clinician. Ultrasound is a safe procedure with very few side effects. In articular joint with no effusion, differentiation of intra-articular from soft tissue injections is difficult. On the other hand, due to variability in pain tolerance and inability to proper positioning of some patients due to severe pain, ultrasound could increase success rate significantly in these situations.

Some previous researches have used clinical improvements as a sign for accuracy of intra-articular injections. In this study, we used fluoroscopy to determine the accuracy of intra-articular injection. It has been shown that several factors have effect on success rate of intraarticular injections in blind approach, such as obesity, BMI, grade of osteoarthritis, needle, expertise of clinician, patient's tolerance to pain and approach to injection. However, when ultrasound is applied success rate would be independent of these factors and this is an advantage to the procedure. Our results showed that blind approach could have a failure rate of $4 \%$ when performed by highly expert clinician close to ultrasound guided injection; however, in hands of inexpert clinician the failure rate could rise sharply to more than $20 \%$ while ultrasound guided failure was not significantly lower than expert clinician. In a study by Sibbitt et al. ${ }^{11}$ they showed that Relative to blind methods, ultrasound guidance resulted in significant (43\%) reduction in procedural pain, improved arthrocentesis success and improved clinical outcomes. ${ }^{10,11}$ Jang et al. ${ }^{12}$ showed that ultrasoundguided intra-articular injections both in-plain and out-of-plain method (97\% success rate) showed significantly higher accuracy rate than injections in the blind injection $\left(78 \%\right.$ success rate).$^{12}$ Park et al. ${ }^{13}$ showed that ultrasound-guided intra-articular injections by long axis in plane approach has a $95 \%$ success rate in consistent with our study. ${ }^{13}$ In another study, ${ }^{14}$ authors showed that US-guided HA injection exhibited a significantly greater accuracy $(96.0 \%)$ than blind injection $(83.7 \%)$ very close to our numbers. In addition to these results, our study showed that unlike blind approach ultrasound has a consistent high success rate independent of the level of expertise of clinician or approach. In a very interesting systematic review, ${ }^{15}$ they showed that ultrasound-guided intra-articular knee injections are more accurate than blinded ones. Blinded injections at SLP site had good accuracy $(87 \%)$ especially if performed by experienced injectors. A systematic review confirmed that accuracy is improved with the use of ultrasound-guided intra-articular injection. ${ }^{16}$

\section{Conclusion}

In conclusion, ultrasound improves accuracy of knee intra-articular injection independent of clinician level of expertise. Blind approaches compose a high failure rate particularly when performed by inexpert clinicians.

\section{Acknowledgments}

None.

\section{Conflicts of interest}

The authors declare there is no conflict of interests.

\section{Funding}

None.

\section{References}

1. Zhang Y, Jordan JM. Epidemiology of osteoarthritis. Clin Geriatr Med. 2010;26(3):355-369.

2. Gerwin N, Hops C, Lucke A. Intraarticular drug delivery in osteoarthritis. Adv Drug Deliv Rev. 2006;58(2):226-242.

3. Felson DT, Lawrence RC, Dieppe PA, et al. Osteoarthritis:new insights. Part 1:the disease and its risk factors. Ann Intern Med. 2000;133(9):726737

4. Hashemi M, Jalili P, Mennati S, et al. The Effects of Prolotherapy With Hypertonic Dextrose Versus Prolozone (Intraarticular Ozone) in Patients With Knee Osteoarthritis. Anesth Pain Med. 2015;5(5):e27585.

5. Reeves KD, Hassanein KM. Long-term effects of dextrose prolotherapy for anterior cruciate ligament laxity. Altern Ther Health Med. 2003;9(3):58-62.

6. Rabago D, Mundt M, Zgierska A, et al. Hypertonic dextrose injection (prolotherapy) for knee osteoarthritis: Long term outcomes. Complement Ther Med. 2015;23(3):388-395.

7. çar D, Diracoglu D, Suleyman T, et al. Intra-articular hyaluronic acid as treatment in elderly and middle-aged patients with knee osteoarthritis. OpUen Rheumatol J. 2013;7(2):38-41.

8. Cohn BT, Shapiro PS. An effective technique for corticosteroid injection into the knee joint. Orthop Rev. 1993;22(12):1341-1342.

9. Hermans J, Bierma-Zeinstra SM, Bos PK, et al. The most accurate approach for intra-articular needle placement in the knee joint:a systematic review. Semin Arthritis Rheum. 2013;43(2):195-203.

10. Sibbitt WL Jr, Peisajovich A, Michael AA, et al. Does sonographic needle guidance affect the clinical outcome of intraarticular injections? J Rheumatol. 2009;36(9):1892-902.

11. Sibbitt WL Jr, Kettwich LG, Band PA, et al. Does ultrasound guidance improve the outcomes of arthrocentesis and corticosteroid injection of the knee? Scand J Rheumatol. 2012;41(1):66-72.

12. Jang SH, Lee SC, Lee JH, et al. Comparison of ultrasound (US)-guided intra-articular injections by in-plain and out-of-plain on medial portal of the knee. Rheumatol Int. 2013;33(8):1951-1959. 
13. Park KD, Ahn JK, Lee SC, et al. Comparison of ultrasound-gui0064ed intra-articular injections by long axis in plane approach on three different sites of the knee. Am J Phys Med Rehabil. 2013;92(11):990-998.

14. Bum Park Y, Ah Choi W, Kim YK, et al. Accuracy of blind versus ultrasound-guided suprapatellar bursal injection. $J$ Clin Ultrasound. 2012;40(1):20-25.
15. Maricar N, Parkes MJ, Callaghan MJ, et al. Where and how to inject the knee-a systematic review. Semin Arthritis Rheum. 2013;43(2):195203

16. Gilliland CA, Salazar LD, Borchers JR. Ultrasound versus anatomic guidance for intra-articular and periarticular injection:a systematic review. Phys Sportsmed. 2011;39(3):121-131. 